\title{
Current and Emerging Drug Therapies in Chronic Lymphocytic Leukemia
}

\author{
Tarsheen K Sethi and Nishitha M Reddy \\ Division of Hematology/Oncology, Department of Medicine, Vanderbilt University Medical Center, Nashville, Tennessee, US
}

DOI: https://doi.org/10.17925/OHR.2017.13.01.34

$\mathrm{U}$

ntil recently, chemoimmunotherapy has been the mainstay of treatment approach in chronic lymphocytic leukemia (CLL) patients requiring intervention. With the emergence of targeted treatments, there has been a shift in CLL therapy. With a better understanding of disease biology and risk stratification, a tailored approach based on patient age and comorbidities has evolved over time. The development of new and potent, next generation CD20 antibodies has refined therapy options especially for elderly unfit patients. Furthermore, agents targeting important pathways involved in proliferation and survival of CLL cells including B-cell receptor (BCR) signaling have provided additional treatment options in traditionally chemo-refractory CLL. Given the rapidly expanding repertoire of drugs, current research is focused on optimizing treatment sequence, duration of treatment and assessing long-term toxicities. Several immune mediated therapies are emerging and new combinations are being tested to re-establish antitumor immune effector response in CLL. While embracing the advances in CLL therapy, a few longstanding lessons remain. There is still little role of treatment of asymptomatic individuals. This review presents an overview of current and emerging drug therapies in the rapidly changing area of CLL treatment.

\section{Keywords}

CLL, novel agents, targeted therapy

Disclosure: Tarsheen K Sethi and Nishitha M Reddy have nothing to disclose in relation to this article. No funding was received for the publication of this article. This study involves a review of the literature and did not involve any studies with human or animal subjects performed by any of the authors.

Authorship: All named authors meet the International Committee of Medical Journal Editors (ICMJE) criteria for authorship of this manuscript, take responsibility for the integrity of the work as a whole, and have given final approval to the version to be published.

open Access: This article is published under the Creative Commons Attribution Noncommercial License, which permits any noncommercial use, distribution, adaptation, and reproduction provided the original author(s) and source are given appropriate credit.

Received: February 10, 2017

Accepted: March 9, 2017

Citation: Oncology \& Hematology Review, 2017;13(1):34-40

Corresponding Author: Nishitha M Reddy, 3927 The Vanderbilt Clinic, Vanderbilt University Medical Center, Nashville, Tennessee, US.

E: nishitha.reddy@vanderbilt.edu
Chronic lymphocytic leukemia (CLL) is the most prevalent adult leukemia with an estimated 18,960 new cases diagnosed in the US in 2016. ${ }^{1}$ The past few years have witnessed major advances in the treatment of CLL with several new drugs receiving US Food and Drug Administration (FDA) approval in both the frontline and relapsed/refractory setting leading to improvement in overall survival (OS). The availability of several active and relatively well-tolerated agents has raised questions on the optimal drug sequence, duration, and tailoring therapy based on the underlying disease biology. With effective drugs in the previously considered poor prognostic individuals, there is also a need to redefine the prognostic criteria. Several guiding principles have already been established including the management of early asymptomatic disease with observation alone. Indications for treatment of untransformed disease include at least one of the following based on The International Workshop Group on CLL (IWCLL) 2008 guidelines: ${ }^{2}$

- Progressive marrow failure due to CLL infiltration as evidenced by worsening cytopenias

- Massive (>6 cm below costal margin) splenomegaly with symptoms

- Bulky (>10 cm) or symptomatic lymphadenopathy

- Progressive lymphocytosis

- Autoimmune cytopenias unresponsive to immunosuppressive therapy

- Presence of B symptoms or significant fatigue (Eastern Cooperative Oncology Group Performance Status [ECOG PS] of 2 or more related to disease).

In those that meet criteria for treatment, important considerations include: patient age, performance status, comorbidities, and presence of chromosomal aberrations. Recent advancements in the understanding of disease biology have highlighted the importance of several potentially targetable pathways contributing to disease pathogenesis such as aberrant activation of BCR signaling pathway, anti-apoptotic BCL2 pathway, and the role of the microenvironment. These novel agents have expanded the repertoire for patients ineligible for chemoimmunotherapy (CIT) due to age or comorbidities and those with poorly responsive disease (high risk genomics such as del[17p]). This review aims to outline the role of standard CIT, targeted agents and ongoing studies of novel agents defining the present landscape of CLL drug treatment. Currently approved therapies and general treatment approach in the first-line and relapsed/refractory (R/R) settings are summarized in Figure 1. 
Figure 1: Approved regimens for chronic lymphocytic leukemia

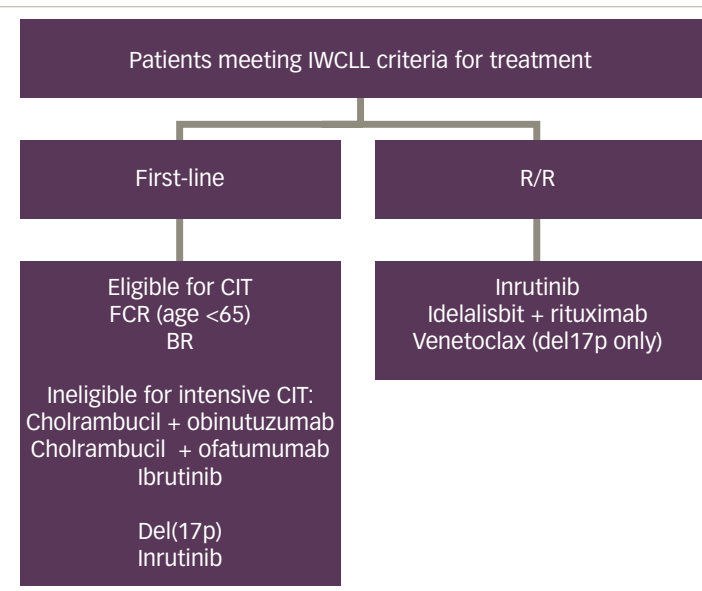

$B R=$ bendamustine, rituximab; $C I T=$ chemoimmunotherapy; $F C R=$ fludarabine, cyclophosphamide, and rituximab; IWCLL = The International Workshop Group on chronic lymphocytic leukemia; $R / R$ = relapsed/refractory.

\section{Chemoimmunotherapy regimens in CLL-fit patients with no major comorbidities}

CIT regimens combine chemotherapeutic agents with a CD20 antibody like rituximab, ofatumumab and obinutuzumab. CIT regimens such as fludarabine, cyclophosphamide, and rituximab (FCR) have been the mainstay of frontline CLL therapy especially in the young, fit individuals. At present, there is no proven benefit to early treatment of patients with CLL. Patients who meet criteria for treatment need to be stratified further based on fitness assessment (age, organ function and performance status) and biological risk (most importantly TP53mutation and /or del[17p]) for treatment decision. Table 1 summarizes the trials comparing different CIT combinations incorporating cytotoxic chemotherapy in combination with a CD20 monoclonal antibody (mAb) that are recommended in the young and elderly fit patients.

FCR is the regimen of choice in young ( $<65$ years), fit patients without evidence of TP53 disruption based on the FCR300 and CLL8 trials. In FCR300, ${ }^{3}$ a single arm phase II study received FCR as first line treatment. At a median follow up of 6 years, complete remission (CR) was seen in $72 \%$ with an overall response rate (ORR) of 95\%. Six-year overall survival (OS) rate was $77 \%$ and progression free survival (PFS) rate was $51 \%$. Recently updated results at a median follow up of 12.8 years show a median PFS of 6.4 years. Considering unmutated (UM) and mutated (M) immunoglobulin heavy chain variable (IGHV) gene status, IGHV-UM and IGHV-M, 12.8 year PFS was $8.7 \%$ and $53.9 \%$ respectively. Importantly, in the IGHV-M subgroup there were no relapses after 10.4 years represented by a plateau on the PFS curve. ${ }^{4} \mathrm{~A}$ larger phase III study, the CLL8 trial compared FCR with FC. FCR was associated with a superior ORR ( $90 \%$ versus $80 \%$ ), CR (44\% versus $22 \%)$, 3-year PFS (65\% versus $45 \%$ ), and 3 -year OS (87\% versus $83 \%)$. FCR was associated with a higher rate of grade $3 / 4$ neutropenia. ${ }^{5}$ Long-term follow up studies after FCR treatment have revealed durable responses in patients with IGHV-M CLL and those without del(17p) or del(11q). ${ }^{4.6,7}$

Bendamustine and rituximab (BR) was evaluated in a phase II study with 117 patients in the front-line setting showed an ORR of $88 \%$ and a CR rate of $23 \%$. At a median follow up of 27 months, the median (m)-PFS was 33.9 months. A quarter of the patients in this study were $>70$ years and their $\mathrm{m}-\mathrm{PFS}$
Table 1: Chemoimmunotherapy regimens in chronic lymphocytic leukemia

\begin{tabular}{|c|c|c|c|c|c|c|}
\hline Regimen & Study/phase & Ref & $\mathrm{n}$ & $\begin{array}{l}\text { ORR } \\
\text { (\%) }\end{array}$ & $\begin{array}{l}\text { CR } \\
(\%)\end{array}$ & PFS (months) \\
\hline \multirow[t]{4}{*}{ FCR } & FCR300/II & 3 & 300 & 95 & 72 & 80 \\
\hline & CLL8/III & 5 & 408 & 90 & 44 & 52 \\
\hline & CLL10/III & 9 & 282 & 95 & 40 & 55 \\
\hline & FCR Lite*/II & 11 & 63 & 94 & 73 & 70 \\
\hline \multirow[t]{2}{*}{$\mathrm{BR}$} & GCLLSG/II & 8 & 117 & 88 & 23 & 34 \\
\hline & CLL 10/III & 9 & 279 & 96 & 31 & 42 \\
\hline PCR & Kay et al/II & 12 & 64 & 91 & 41 & 33 \\
\hline O-FC & Wierda et al/II & 13 & 61 & 75 & 41 & NR (8 month follow up) \\
\hline O-PC & Shanafelt et al/II & 14 & 48 & 96 & 46 & Not reported \\
\hline G-FC & Galton/lb & 10 & 21 & 62 & 24 & NR (24 month follow up) \\
\hline G-B & Galton/Ib & 10 & 20 & 90 & 45 & NR (24 month follow up) \\
\hline $\mathrm{O}-\mathrm{C}$ & Complement1/III & 16 & 447 & 82 & 14 & 22 \\
\hline $\mathrm{G}-\mathrm{Clb}$ & CLL11/III & 15 & 333 & 78 & 20 & 27 \\
\hline
\end{tabular}

$B R=$ bendamustine, rituximab; $C L L=$ chronic lymphocytic leukemia; $C R=$ complete remission; FCR = fludarabine, cyclophosphamide, and rituximab; $G-B=$ obinutuzumab and bendamustine; G-Clb = obinutuzumab and chlorambucil; $G-F C=$ obinutuzumab, fludarabine and cyclophosphamide; $N R=$ not reached; $O-C=$ chlorambucil and ofatumumab; O-FC = fludarabine, cyclophosphamide, and ofatumumab;

$O-P C=$ pentostatin, cyclophosphamide, and ofatumumab; ORR = overall response rate; $P C R=$ pentostatin, cyclophosphamide, and rituximab; $P F S=$ progression free survival; Ref = reference.

was similar to younger patients despite a lower ORR in this age group. ${ }^{8}$ Further in the randomized phase III CLL10 trial, BR was compared with FCR in the front-line setting. This was a non-inferiority trial with PFS as primary endpoint and excluded patients with del(17p). The final analysis of 561 patients included 282 patients in the FCR arm and 279 in the BR arm. At a median follow up of 37.1 months, the m-PFS was 55.2 months and 41.7 months in the FCR and BR arms respectively. OS was not different between the two arms. The FCR arm was associated with more grade $3 / 4$ neutropenia and infections especially in patients older than 65 years. There was no difference in PFS in the subgroup of patients $>65$ years. In addition, post-hoc analysis, favored the use of FCR in patients with IGHV-UM ( $p=0.017)$ and del(11q) ( $p=0.0002) \mathrm{CLL}$. Due to a lower rate of complications in older patients, BR is a reasonable front-line choice in fit CIT eligible patients $>65$ years of age. Several additional CIT regimens were tested in the frontline setting and are summarized in Table 1.10-14

\section{Less intensive regimens-older patients with comorbidities}

For elderly or patients, otherwise unfit for the above mentioned "intensive" CIT regimens and in the absence of del(17p), several different options have emerged in the past few years as frontline therapy. The current standard regimen in this setting is the combination of chlorambucil with an anti-CD20 antibody. ${ }^{15} \mathrm{~A}$ phase III (COMPLEMENT-1) study included 447 patients who were $\geq 65$ years of age and evaluated chlorambucil versus chlorambucil + ofatumumab (O-C). At a median follow up of 28.9 months, the m-PFS was 22.4 months in the combination arm, versus 13.1 months in the chlorambucil arm. The ORR and CR rates were $82 \%$ versus $69 \%$, and $14 \%$ versus $1 \%$ respectively favoring the combination. ${ }^{16}$ The study however, lacks head-to-head comparison with rituximab likely because at the time of trial design, chlorambucil as a single agent was an acceptable comparator. 
Table 2: Targeted therapies in chronic lymphocytic leukemia-ongoing and completed studies

\begin{tabular}{|c|c|c|c|c|c|c|}
\hline Agent/combination & Study/phase & Line & Ref & ORR (\%) & PFS (mo) & Trial Number \\
\hline \multirow[t]{2}{*}{ Ibrutinib } & RESONATE/III & RR & 29,30 & 63 & NR at 9 mo & \\
\hline & RESONATE 2/III & $\mathrm{F}$ & 33 & 86 & NR at $18 \mathrm{mo}$ & \\
\hline Ibutinib + BR & HELIOS/III & RR & 35 & 83 & NR at $17 \mathrm{mo}$ & \\
\hline Ibritutinib + obinutuzumab & ILLUMINATE/III & $\mathrm{F}$ & & & & NCT02264574 \\
\hline Ibritutinib + rituximab & ECOG-E1912 /III & $\mathrm{F}$ & & & & NCT02048813 \\
\hline Ibrutinib + rituximab & $\begin{array}{l}\text { ALLIANCE } \\
\text { A041202/III }\end{array}$ & $\mathrm{F}$ & & & & NCT01886872 \\
\hline Acalabrutinib (ACP-196) & 1/II & RR & 36 & 95 & NR at $14 \mathrm{mo}$ & \\
\hline ACP-196 versus ACP-196 + obinutuzumab & ACE-CL-007/III & $\mathrm{F}$ & & & & NCT02475681 \\
\hline ACP-196 versus ibrutinib & ACE-CL-006/III & RR & & & & NCT02477696 \\
\hline Idelalisib + rituximab & III & RR & 44 & 81 & & \\
\hline Idelalisib/obinutuzumab & GS-US-312-0118/III & $\mathrm{F}$ & & & & NCT01980875 \\
\hline BR + idelalisib & GS-US-312-0123 & $\mathrm{F}$ & & & & NCT01980888 \\
\hline Venetoclax & 1 & RR & 40 & 79 & & \\
\hline Venetoclax + obinutuzumab & III & $\mathrm{F}$ & & & & NCT02242942 \\
\hline Venetoclax + rituximab & III & RR & & & & NCT02005471 \\
\hline
\end{tabular}

$B R=$ bendamustine, rituximab; $F=$ first-line; $m o=$ months; $N R=$ not reached; $O R R=$ overall response rate; $P F S=$ progression free survival; $R$ ef $=$ reference; $R R=$ relapsed $/$ refractory.

The phase III CLL11 trial randomized 780 patients to chlorambucil alone versus chlorambucil + rituximab $(\mathrm{R}-\mathrm{C})$ versus $\mathrm{O}-\mathrm{C}$ in a 1:2:2 fashion. The O-C arm was superior to R-C in terms of PFS (median, 26.7 versus 16.3 months), ORR (78.4\% versus $65.1 \%)$, CR (20.7\% versus $7.0 \%)$, and minimal residual disease (MRD). ${ }^{15}$ Infusion related reactions were notably higher in patients receiving O-C, $20 \%$ as compared with $4 \%$ in the R-C arm. Based on these two trials, the combination of chlorambucil with ofatumumab or obinutuzumab are FDA approved for front-line therapy of CLL patients.

\section{Therapies directed against very-high risk CLL in the pre-kinase era}

Patients with very-high risk CLL such as those with del(17p) or TP53 gene mutation have poor response to the above mentioned chemotherapy as such agents are dependent on the DNA damage pathway for their cytotoxicity. ${ }^{3,5,17}$ For example, the CR rate with $\mathrm{FCR}$ in this high risk population in the CLL8 trial was $5 \%$ with a m-PFS of 11.3 months. ${ }^{5}$ Similarly, in patients treated with BR the m-PFS was 7.9 months with no patient achieving a CR. ${ }^{8}$ Alemtuzumab, an anti-CD52 antibody was evaluated in CLL as first-line therapy in a phase III trial but failed to improve response in del(17p) patients with a median PFS of 10.7 months in this population. ${ }^{18}$ The combination of alemtuzumab and steroids was evaluated further in the CLL206 trial with a m-PFS of 18.3 months and in another trial with this combination (CLL20), the m-PFS was 32.8 months. The improved outcome was attributed to the use of allogeneic stem cell transplantation as consolidation. 19,20

The combination of Rituximab with high dose methylprednisolone (HDMP) was tested in patients with fludarabine refractory disease and found to have an ORR of 93\% with 36\% attaining CR. ${ }^{21}$ The dosing schedule was three cycles of rituximab ( $375 \mathrm{mg} / \mathrm{m}^{2}$ weekly for 4 weeks) in combination with HDMP ( $1 \mathrm{gm} / \mathrm{m}^{2}$ daily for 5 days). While this regimen was associated significant steroid-related side effects including fluid retention and transient hyperglycemia, it was minimally myelosuppressive making it an option in otherwise high risk and/or elderly patients. This combination was tested in the front line setting in 28 patients, with 3 days of HDMP to minimize toxicities. ${ }^{22}$ The ORR was $96 \%$ and CR was $32 \%$. The m-PFS at 3-year follow up was 30 months. While these two studies included few patients with del(17p), Pileckyte et al. reported their results with this combination in R/R high risk CLL with 29 patients including 13 with del(17p). The ORR in del(17p) in the R/R setting was 69\%. The regimen used in this study was more intense with 5 days of HDMP and each cycle had two doses of Rituximab up to a total of six cycles. ${ }^{23}$ Overall, with these studies, the possibility of using rituximab with HDMP is an option in the high-risk cytogenetics group especially in those with poor bone marrow reserve.

\section{Therapies targeting the CLL-signaling pathways}

The B-cell receptor (BCR) is important in the pathogenesis of CLL mediating the signal for cell division. Furthermore, the differential responsiveness of $\mathrm{BCR}$ to stimuli is implicated in the differences in prognosis of IGHV-M and IGHV-UM. ${ }^{24}$ Apart from BCR signaling, the BCL2 pathway has become a target in CLL. The effectiveness of these agents in patients with del 17p or TP53 mutations has been encouraging. Table 2 summarizes completed and ongoing studies of targeted therapies in CLL.

\section{Bruton tyrosine kinase inhibitors}

Bruton tyrosine kinase (BTK) is a non-receptor tyrosine kinase that belongs to Tec family of kinases. It has an important role in BCR signaling leading to downstream activation of pathways important for CLL cell survival including NF- $\mathrm{BB}$ and PI3K. It also contributes to CLL pathogenesis by being involved in $\mathrm{B}$-cell migration and adhesion. ${ }^{25-27} \mathrm{Ibrutinib}$ is an oral selective and irreversible inhibitor of BTK that acts by forming a bond with cysteine-481 of BTK. ${ }^{28}$ Ibrutinib is FDA approved for patients with R/R CLL and those with del(17p) CLL. In a large phase III (RESONATE) trial in 391 patients (127 harbored a del[17p] with $\mathrm{R} / \mathrm{R} \mathrm{CLL}$, ibrutinib was associated with a $63 \%$ response rate [70\% investigator assessed]). At a median follow up of 9.4 months, the PFS was not reached in the ibrutinib arm and 8.1 months in the ofatumumab arm. The 1 year OS was $90 \%$ in the ibrutinib arm and $81 \%$ in the ofatumumab arm. ${ }^{29,30}$ In a small phase $1 /$ II study in elderly patients with a median age of 
71 years, O'Brien et al found an ORR of 71\% (95\% confidence interval [CI] 52.0-85.8) with $13 \%$ CR rate at a median follow up of 22 months. Six percent of patients had del(17p). ${ }^{31}$ At 3-year follow up, the ORR was $84 \%$, with $23 \%$ attaining CR. The 30 -month PFS was $96 \%$, and OS was $97 \% .{ }^{30}$ Another phase III study evaluated response of ibrutinib in 51 patients with disruption in TP53 pathway (47 with del(17p) and 4 with TP53 mutation without del(17p) including 35 patients with previously untreated CLL). The ORR in previously untreated patients with $97 \%$ and R/R CLL patients was $80 \%$. The CR rate in untreated patients was $12 \%$ with a median time to CR of 48 weeks and 2 year PFS was $91 \% .{ }^{32}$ These studies have led to the acceptance of ibrutinib as first line treatment agent of choice in CLL patients with TP53 mutation and/or del(17p). RESONATE 2 is a phase III trial that tested ibrutinib as first line treatment in patients 65 years or older without del(17p). Treatment naiive patients, requiring therapy were randomized to receive ibrutinib or chlorambucil. At a median follow up of 18.4 months, the ORR was $86 \%$ in the ibrutinib arm compared with $35 \%$ in the chlorambucil arm. Ibrutinib resulted in a higher m-PFS (not reached versus 19 months) and 24-month OS $(98 \%$ versus $85 \%){ }^{33}$ Lymphocytosis after the initiation of treatment with BTK inhibitors including ibrutinib is common and self-limited, often resolving within 6-9 months of starting treatment. It is not associated with any clinical adverse effects or adverse clinical outcome. ${ }^{34}$

In a recent study, CIT (BR) was compared with bendamustine, rituximab, and ibrutinib in a phase III trial (HELIOS). The ORR was $83 \%$ compared with $68 \%$ in the group treated with BR alone. At the time of analysis $79 \%$ of patients receiving Ibrutinib were in remission versus only $24 \%$ in patients who did not. At a median follow up of 17 months, the m-PFS was not reached in patients receiving ibrutinib with $\mathrm{CIT}$ whereas the $\mathrm{m}$-PFS was 13.2 months in patients treated with BR. ${ }^{35}$ The major critique concerning this trial was the question if chemotherapy added any benefit to ibrutinib, as the combination therapy yielded similar results that were comparable to single agent ibrutinib in prior studies. In an ongoing frontline CLL phase III trial in elderly patients ( $>65$ years), otherwise ineligible for chemotherapy are being randomized to receive either obinutuzumab and ibrutinib or obinutuzumab and chlorambucil (ILLUMINATE study; NCT02264574). Other studies evaluating ibrutinib combinations include untreated CLL/SLL randomized to either FCR or ibrutinib and rituximab in a phase III trial (NCT02048813); and NCT01886872, a multi-arm phase III trial with Ibrutinib alone, Ibrutinib and rituximab or BR in older patients in the front-line setting.

Acalabrutinib (ACP-196) is a second-generation, selective, irreversible inhibitor of BTK. It has rapid oral absorption and higher selectivity with the absence of irreversible inhibition of off target kinases, such as epidermal growth factor receptor (EGFR), tyrosine kinase expressed in hepatocellular carcinoma (TEC), and interleukin-2-inducible T-cell kinase (ITK). In a study of 60 patients, at a median follow-up of 14.3 months, the ORR overall response was $95 \%$ with $5 \%$ having stable disease. Thirty percent of patients had del(17p) and experienced an ORR of 100\%. Most common side effects were headache, diarrhea, and weight gain and there were no cases of atrial fibrillation have been reported to date. ${ }^{36}$ Ongoing phase III trials include acalabrutinib in isolation or combination in the first-line (NCT02475681) and in R/R CLL (NCT02029443; NCT02477696). Other novel BTK inhibitors in development include ONO-4059 which was well tolerated in its phase I study and has an ORR of $84 \%$ across the board including in those with del(17p). ${ }^{37}$ CC-292 (spebrutinib) showed an ORR of $63 \%$ in its phase I study. ${ }^{38}$
Figure 2: Targets of therapy in the B-cell receptor pathway and selected other targets in CLL therapy

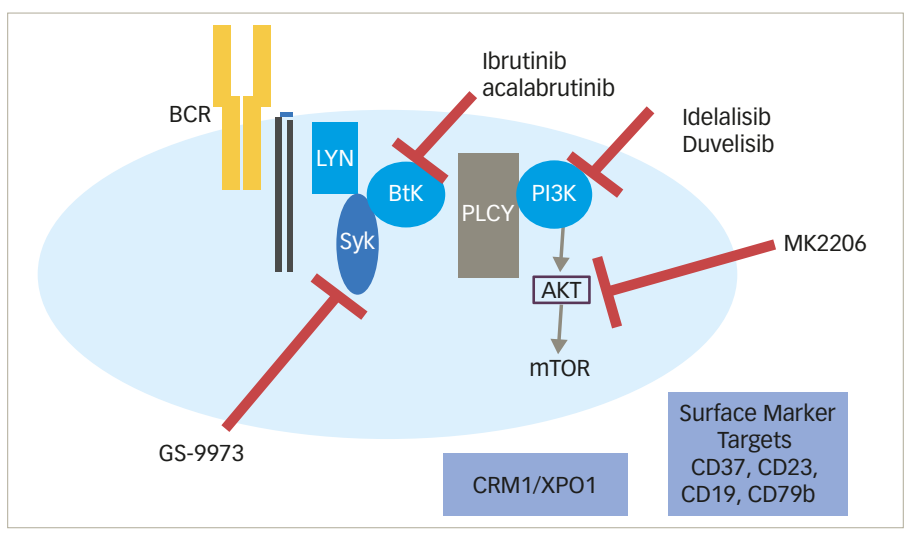

$A K T=$ protein kinase $B ; B C R=B$-cell receptor; $B t K=$ Bruton's tyrosine kinase; $L Y N=$ tyrosine protein kinase lyn; $m T O R=$ mechanistic target of rapamycin; $P L C Y=$ phosphoinositoide phospholipase $C$; syk = spleen tyrosine kinase.

\section{BCL2 inhibitors in CLL}

Like several other hematologic malignancies, BCL2 pro-survival protein family is over expressed in CLL and mediates CLL cell survival. This led to the development of BH3 mimetic agents that antagonize BCL2..$^{39}$ Venetoclax is an oral, selective BCL2 inhibitor. It is FDA approved for treatment of R/R del(17p) CLL. As monotherapy, venetoclax showed an ORR of 79\% and CR rate of $20 \%$ at a median follow up of 15 months. Median PFS was 18 months. ${ }^{40}$ In a phase II multicenter study of 107 relapsed patients harboring del(17p). At a median follow-up of 12.1 months, ORR was $79.4 \%{ }^{41}$ The most common grade $3 / 4$ adverse events were neutropenia $(40 \%)$, infection (20\%), anemia (18\%), and thrombocytopenia (15\%). Given the risk for tumor lysis syndrome, the drug is administered via a weekly dose ramp-up schedule over 4-5 weeks followed by a continuous dosing of $400 \mathrm{mg} /$ day until disease progression. In combination with rituximab, at a median follow-up of 28 months, the ORR was $86 \%$, including a complete response in $51 \%$ of patients. Fifty-seven percent of patients had negative MRD. ${ }^{41}$ With these impressive results in R/R CLL, venetoclax is currently being studied in the front line setting where patients with $C L L$ and a high Cumulative IIIness Rating Scale (CIRS) score are being randomized to venetoclax + obinutuzumab or O-C (NCT02242942). In patients with $\mathrm{R} / \mathrm{R} \mathrm{CLL}$, a phase III trial comparing venetoclax + rituximab versus BR is ongoing (NCT02005471). Based on preclinical evidence of synergy, a phase II study evaluating venetoclax and ibrutinib in R/R or untreated high risk CLL is ongoing (NCT02756897). ${ }^{42}$

\section{PI3 kinase inhibitors}

PI3K pathway is involved in normal B cell activation and survival. Hyperactivity of this pathway plays a role in the pathogenesis of several B cell malignancies including CLL. ${ }^{43}$ idelalisib is an oral selective PI3Kס inhibitor that has activity in CLL and is approved in the R/R CLL setting in combination with rituximab. In a multicenter phase III study, idelalisib and rituximab was compared with rituximab alone. Patients receiving combination therapy had improved rates of overall response $(81 \%)$, and OS at 12 months ( $92 \%$ versus $80 \% \mathrm{p}=0.02) .{ }^{44}$ The most commonly reported adverse events reported include; fatigue (24\%), diarrhea (19\%), transaminitis (35\%). Neutropenia (3\%), and pneumonitis (4\%) were other serious adverse events. In a prespecified subgroup analysis, patients with either 17p deletion or TP53 mutation had improved PFS with idelalisib 
and rituximab. Data in the frontline setting is limited. Ongoing frontline combination therapies that included idelalisib were halted due to concern for toxicities.

Several other agents that target the PI3Kinase pathway are currently under evaluation. Duvelisib (IPI-145), a novel oral PI3K $\delta$ and PI3K-Y inhibitor was evaluated in a phase I study in R/R CLL patients. The rate of grade $3 / 4$ neutropenia was $42 \%$ and transaminitis of $9 \%$, while the ORR was $58 \%$ with $2 \%$ of the 55 patients achieving a CR. ${ }^{45}$ While duvelisib was seen to overcome ibrutinib resistance due to C481S mutation and has been seen to have activity in ibrutinib refractory disease, its recent development as a CLL agent has been paused by its source pharmaceutical company.46-48 TGR1202 is a second generation oral PI3K $\delta$ inhibitor being tested in non-Hodgkin Iymphoma (NHL) including CLL, in $20 \mathrm{CLL}$ patients the ORR was 63\%. Most common grade $3 / 4$ toxicities were cytopenias, dyspnea, rash, and fatigue. There was lower incidence of transaminitis, colitis, and pneumonia. ${ }^{49}$ It has been studied in combination with ublituximab, a novel, chimeric mAb targeting a unique epitope on the CD20 antigen in R/R CLL, an ORR of 83\% was seen ${ }^{50} \mathrm{Pl} 3-$ Kinase inhibitors are effective in CLL therapy. Their unique toxicities although concerning and may have been associated with early treatment discontinuation, vigilance in symptom monitoring an appropriate management is prudent for the success of this therapy.

\section{Immunomodulators and other immune based treatments in CLL}

Lenalidomide is by far the most common immunomodulator (IMiD) that has been studied in CLL. It was found to possess moderate activity in R/R CLL. ${ }^{51-53}$ Badoux et al. reported outcome on 60 patients (>65 years). At a median follow up of 29 months, the ORR was $65 \%$ with a CR rate of $10 \%$. Estimated 2-year PFS was 60\%. The starting dose in this study was $5 \mathrm{mg}$ / day with an escalation by $5 \mathrm{mg} / \mathrm{cycle}$ up to a maximum of $25 \mathrm{mg}$. The median dose in this study was $5 \mathrm{mg} /$ day. ${ }^{54}$ Chen et al. reported outcome on 25 patients with a median age of 60 years. With the initial starting dose of $10 \mathrm{mg}$, two patients had serious adverse events including tumor lysis and sepsis. The protocol was amended to have a starting dose of $2.5 \mathrm{mg}$ up to a target dose of $10 \mathrm{mg}$, allowing escalation up to $25 \mathrm{mg}$ in non-responders. The median dose administered was $15 \mathrm{mg}$. ORR seen in this study was $56 \%$. In the follow-up analysis, the ORR was $72 \%$ with a CR of $20 \%$. The 3-year PFS was $65 \%$ and OD was $85 \% .55,56$ Treatment with lenalidomide is associated with tumor flare in more than half of the patients and this is clinically seen as a sudden enlargement of lymph nodes accompanied by redness and tenderness. This change is benign and attributed to an "immune activation" response. ${ }^{57}$ Ongoing combination studies of lenalidomide include those with obinutuzumab (NCT02225275, NCT02371590) and a triple combination with rituximab and ibrutinib in R/R CLL (NCT02200848). Although active, lenalidomide does not have an FDA approved indication in CLL therapy. Lenalidomide has been evaluated in the maintenance setting in newly diagnosed high risk patients following CIT with an improvement in PFS. ${ }^{58}$

Ramsay et al. demonstrated that T cells from CLL patients have higher expression of inhibitory immune checkpoint molecules such as cytotoxic T-lymphocyte-associated protein 4 (CTLA-4) and programmed cell death protein-1 (PD-1) and have impaired immune synapse function and thereby reduced cytotoxicity. CLL cells express the corresponding ligands both PDL1 and PD-L2. ${ }^{59-61}$ Furthermore, there is evidence for $\mathrm{T}$ cell exhaustion in CLL. ${ }^{62}$ Preclinical studies in a T-Cell leukemia/lymphoma protein 1 (TCL-
1) mouse model have shown evidence of the efficacy of PD-L1 blockade in mice where this restored the immune synapse function and prevented the development of CLL with early intervention. Several clinical trials are examining the role of this in humans (NCT02420912, NCT02332980). Based on preclinical evidence of synergy with Ibrutinib, Ongoing trials are examining the role of this combination in CLL. Jain et al. presented the preliminary results of this combination in R/R CLL or Richter transformation in 12 patients with evidence of activity in this patient population. ${ }^{63}$

A detailed discussion of CAR-T cell therapy in CLL is beyond the scope of this review but briefly, CD-19 CAR-T cell therapy has been found to have an ORR of $45-57 \%$ in various studies. ${ }^{64,65}$

\section{Novel pathways and drugs}

Beyond immunotherapies, novel pathways of interest have gained interest as targets for CLL therapy. Antibodies against cell surface markers such as CD37, CD23, CD19, CD79b, and selective inhibitors of nuclear exporter of tumor suppressors, CRM1/XPO1, are under evaluation in clinical studies based on preclinical data.66-74 Stimulation of the BCR results in activation of the associated spleen tyrosine kinase (Syk), which mediates downstream signaling, and amplification of response. Syk is therefore a viable target and GS-9973 (entospletinib) is under development as a selective Syk inhibitor. ${ }^{75,76}$ A phase II study in R/R CLL showed an ORR of 61\%, and serious adverse events included dyspnea, pneumonia, fever and febrile neutropenia76 CD37 is a cell surface marker seen on mature B cells and is expressed on CLL cells at a high level. ${ }^{74}$ Otlertuzumab (TRU-016), a novel humanized anti-CD37 protein has been studied in a phase I study in R/R CLL and also in combination with rituximab. ${ }^{77,78}$ An anti-CD37 antibody-drug conjugate IMGN529 is being studied in R/R CLL in a phase I study (NCT01534715). ${ }^{79}$ Another CD37 directed therapy is $\mathrm{BI} 836826$ an anti-CD37 mAb which is being studied in a phase I study (NCT01296932). ${ }^{80}$ Another cell surface target CD23 is expressed on CLL cells and functions as an immunoglobulin E (IgE) receptor. Drugs targeting $C D 23$ include a $\mathrm{mAB}$, lumiliximab, that failed to show added benefit when combined with FCR. ${ }^{81}$ MEDI-551 is a CD19 antibody that has been tested in R/R CHL and there are ongoing studies in combination with bendamustine (NCT01466153). Another CD19 mAb XmAb5574 (MOR208) with activity in a phase I study, is being tested in combination with lenalidomide (NCT02005289). ${ }^{82}$ Based on preclinical evidence of activity, ${ }^{83}$ selective inhibitors of nuclear export that target XPO-1, which showed activity in CLL in a phase I study, is being tested in combination with ibrutinib in R/R CLL (NCT02303392). Preclinical data suggested that the CXCR4/CXCL12 axis is important in CLL and plays a role in the homing and retention of the malignant CLL cell in tissues including the bone marrow. Small molecule CXCR antagonist, plerixafor was hypothesized to improve the sensitivity to rituximab by mobilizing CLL cells and was tested in the R/R setting (NCT00694590). Preliminary results from this phase I study showed that the combination was well tolerated and showed an ORR of $36 \%$ (all partial response) in 14 patients. $^{84}$ In addition to these drugs under study in clinical trials in CLL, preclinical studies have outlines several additional pathways that can be targeted for therapy including CD38, nuclear factor-кB and MYD88 (beyond lenalidomide and ibrutinib), surrogates of zeta chainassociated protein kinase 70 (ZAP 70), TCL-1, induced myeloid leukemia cell differentiation protein ( $\mathrm{MCl}-1)$, CD49d (possibly also affected by ibrutinib and idelalisib), translocation-associated notch protein (TAN-1) (NOTCH1), and Splicing factor 3B1 (SF3B1). ${ }^{85}$ 


\section{Evolving cost burden of CLL with emerging therapies}

As targeted therapies are being used both in the first-line and relapsed CLL setting and resulting in longer survival in many patients, with prolonged duration of therapy, it is important to acknowledge the economic impact of these advances in treatment. Using a Markov micro-simulation prediction model, Jain et al. outlined the projected increase in prevalence and total cost of CLL treatment from 2011-2035.86 They predict that compared with \$0.9 billion in 2011, the annual cost of CLL treatment is projected to increase to $\$ 3.5$ billion in 2025. Shanafelt et al. have raised similar issues in this context taking into account the cost burden of ibrutinib and idelalisib therapy. ${ }^{87}$ This is of concern as this can potentially affect the access and adherence to the prescribed drugs and raises the need for sustainable pricing of these drugs.

\section{Conclusion}

In view of the fortunate advances in the field of $C L L$, the treatment algorithms in this disease are rapidly changing. These advancements have led to a change in the challenges of CLL treatment from having too few options to now deciding on the optimal sequencing and duration of the many available options. When available, participation in clinical trials should be considered in both front-line and R/R CLL. With several approved therapies in hand, patient factors such as: performance status, comorbidities, cytogenetic risk, and toxicity profile of drugs should be taken into consideration before deciding on the best option. At present, there is no role of starting treatment in the asymptomatic patients and criteria from IWCLL 2008 guidelines remain important in deciding who needs treatment. $\square$
1. Siegel RL, Miller KD, Jemal A, Cancer statistics, 2016, CA Cancer Clin, 2016;66:7-30.

2. Hallek M, Cheson BD, Catovsky D, et al., Guidelines for the diagnosis and treatment of chronic lymphocytic leukemia: a report from the International Workshop on Chronic Lymphocytic Leukemia updating the National Cancer Institute-Working Group 1996 guidelines, Blood, 2008:111:5446-56.

3. Tam CS, O'Brien S, Wierda W, et al., Long-term results of the fludarabine, cyclophosphamide, and rituximab regimen as initial therapy of chronic lymphocytic leukemia, Blood, 2008;112:975-80. . Thompson PA, Tam CS, O'Brien SM, et al., Fludarabine, cyclophosphamide, and rituximab treatment achieves long-term disease-free survival in IGHV-mutated chronic lymphocytic leukemia, Blood, 2016:127:303-9.

5. Hallek M, Fischer K, Fingerle-Rowson $G$, et al., Addition of rituximab to fludarabine and cyclophosphamide in patients with chronic lymphocytic leukaemia: a randomised, open-label, phase 3 trial, Lancet, 2010;376:1164-74.

6. Rossi D, Terzi-di-Bergamo L, De Paoli L et al., Molecular prediction of durable remission after first-line fludarabinecyclophosphamide-rituximab in chronic lymphocytic leukemia, Blood, 2015;126:1921-4.

7. Fischer K, Bahlo J, Fink AM, et al., Long term remissions after FCR chemoimmunotherapy in previously untreated patients with $\mathrm{CL}$. updated results of the CLL8 trial, Blood, 2015:127:208-15

8. Fischer K, Cramer P, Busch R, et al., Bendamustine in combination with rituximab for previously untreated patients with chronic lymphocytic leukemia: a multicenter phase II trial of the German Chronic Lymphocytic Leukemia Study Group, I Clin Oncol, 2012;30:3209-16.

9. Eichhorst B, Fink A-M, Bahlo J, et al., First-line chemoimmunotherapy with bendamustine and rituximab versus fludarabine, cyclophosphamide, and rituximab in patients with advanced chronic lymphocytic leukaemia (CLL10): an international, open-label, randomised, phase 3 , non-inferiority trial, Lancet Oncol, 2016;17:928-42.

10. Brown JR, O'Brien S, Kingsley CD, et al., Obinutuzumab plus fludarabine/cyclophosphamide or bendamustine in the initial therapy of CLL patients: the phase 1b GALTON trial, Blood, 2015;125:2779-85.

11. Foon KA, Mehta D, Lentzsch $S$, et al., Long-term results of chemoimmunotherapy with low-dose fludarabine, cyclophosphamide and high-dose rituximab as initial treatment for patients with chronic lymphocytic leukemia, Blood, 2012;119:3184-5

12. Kay NE, Geyer SM, Call TG, et al., Combination chemoimmunotherapy with pentostatin, cyclophosphamide, and rituximab shows significant clinical activity with low accompanying toxicity in previously untreated $\mathrm{B}$ chronic accompanying toxicity in previously untreated B
lymphocytic leukemia, Blood, 2007:109:405-11.

13. Wierda WG, Kipps TJ, Durig J, et al., Chemoimmunotherapy with $\mathrm{O}-\mathrm{FC}$ in previously untreated patients with chronic lymphocytic leukemia Blood 2011;117:6450-8

14. Shanafelt T, Lanasa MC, Call TG, et al., Ofatumumab-based chemoimmunotherapy is effective and well tolerated in patients with previously untreated chronic lymphocytic leukemia (CLL), Cancer, 2013:119:3788-96.

15. Goede V, Fischer K, Busch R, et al., Obinutuzumab plus chlorambucil in patients with CLL and coexisting conditions, N Eng/ J Med, 2014;370:1101-10

16. Hillmen P, Robak T, Janssens $A$, et al., Chlorambucil plus ofatumumab versus chlorambucil alone in previously untreated patients with chronic lymphocytic leukaemia (COMPLEMENT 1): a randomised, multicentre, open-label phase 3 trial, Lancet, 2015:385:1873-83.

17. Zenz T, Eichhorst B, Busch $R$, et al., TP53 mutation and survival in chronic lymphocytic leukemia, J Clin Oncol, 2010;28:4473-9.

18. Hillmen P, Skotnicki AB, Robak T, et al., Alemtuzumab compared with chlorambucil as first-line therapy for chronic lymphocytic leukemia, J Clin Oncol, 2007;25:5616-23.

19. Pettitt AR, Jackson R, Carruthers $S$, et al., Alemtuzumab in combination with methylprednisolone is a highly effective induction regimen for patients with chronic lymphocytic leukemia and deletion of TP53: final results of the national cancer research institute CU 206 trial, J Clin Oncol, 2012:30:1647-55.

20. Stilgenbauer S, Cymbalista F, Leblond V, et al., Alemtuzumab combined with dexamethasone, followed by alemtuzumab maintenance or Allo-SCT in "ultra high-risk" CLL: final results from the CLL2O phase II study, Blood, 2014:124:1991.

21. Castro JE, Sandoval-SuS JD, Bole J, et al., Rituximab in combination with high-dose methylprednisolone for the treatment of fludarabine refractory high-risk chronic lymphocytic leukemia, Leukemia, 2008:22:2048-53.

22. Castro JE, James DF, Sandoval-Sus JD, et al., Rituximab in combination with high-dose methylprednisolone for the treatment of chronic lymphocytic leukemia, Leukemia 2009;23:1779-89

23. Pileckyte $R$, Jurgutis $M$, Valceckiene $V$, et al., Dose-dense highdose methylprednisolone and rituximab in the treatment of relapsed or refractory high-risk chronic lymphocytic leukemia Leuk Lymphoma, 2011:52:1055-65.

24. Stevenson FK, Caligaris-Cappio F, Chronic lymphocytic leukemia: revelations from the B-cell receptor, Blood, 2004;103:4389-95

5. Ponader S, Burger JA, Bruton's tyrosine kinase: from X-linked agammaglobulinemia toward targeted therapy for B-cell malignancies, J Clin Oncol, 2014:32:1830-9.

26. Herman SEM, Gordon AL, Hertlein E, et al., Bruton tyrosine kinase represents a promising therapeutic target for treatment of chronic lymphocytic leukemia and is effectively targeted by PCl-32765, Blood, 2011:117:6287-96.

27. Satterthwaite $A B$, The role of Bruton's tyrosine kinase in B-cell development and function: a genetic perspective, Immunol Rev, 2000;175:120-7.

28. Pan Z, Scheerens H, Li SJ, et al., Discovery of selective irreversible inhibitors for Bruton's tyrosine kinase, ChemMedChem, 2007:2:58-61.

29. Byrd JC, Brown JR, O'Brien S, et al., Ibrutinib versus ofatumumab in previously treated chronic lymphoid leukemia, N Engl J Med 2014;371:213-23

30. Byrd JC, Furman RR, Coutre SE, et al., Three-year follow-up of treatment-naive and previously treated patients with $C L L$ and $S L$ receiving single-agent ibrutinib, Blood, 2015;125:2497-506

31. O'Brien S, Furman RR, Coutre SE, et al., Ibrutinib as initial therapy for elderly patients with chronic lymphocytic leukaemia or small Iymphocytic lymphoma: an open-label, multicentre, phase 1b/2 trial, Lancet Oncol, 2014:15:48-58.

32. Farooqui MZH, Valdez J, Martyr S, et al., Ibrutinib for previously untreated and relapsed or refractory chronic lymphocytic leukaemia with TP53 aberrations: a phase 2, single-arm tria , Lancet Oncol, 2015;16:169-76.

33. Burger JA, Tedeschi A, Barr PM, et al. Ibrutinib as initial therapy for patients with chronic lymphocytic leukemia, N Engl J Med, 2015:373:2425-37.

34. Woyach JA, Smucker K, Smith LL, et al., Prolonged lymphocytosis during ibrutinib therapy is associated with distinct molecula characteristics and does not indicate a suboptimal response to therapy, Blood, 2014;123:1810-7.

35. Chanan-Khan A, Cramer P, Demirkan F, et al., Ibrutinib combined with bendamustine and rituximab compared with placebo, bendamustine, and rituximab for previously treated chronic lymphocytic leukaemia or small lymphocytic lymphoma (HELIOS): a randomised, double-blind, phase 3 study, Lancet Oncol, 2016:17:200-11.

36. Byrd JC, Harrington B, O'Brien S, et al., Acalabrutinib (ACP-196) in relapsed chronic lymphocytic leukemia, N Eng/ I Med, 2016;374:323-32

37. Fegan C, Bagshawe J, Salles G, et al., The Bruton's tyrosine kinase (BTK) inhibitor ONO-4059: promising single agent activity and wel tolerated in patients with high risk chronic lymphocytic leukaemia (CLL), Blood, 2014:124:3328.

38. Harb WA Hill BT Gabrilove 1 et al., Phase 1 study of single agent CC-292, a highly selective Bruton's tyrosine kinase (BTK) inhibitor in relapsed/refractory chronic lymphocytic leukemia (CLL), Blood 2013;122:1630

39. Anderson MA, Huang D, Roberts A, Targeting BCL2 for the treatment of lymphoid malignancies, Semin Hematol 2014; $51: 219-27$

40. Roberts AW, Davids MS, Pagel JM, et al., Targeting BCL2 with venetoclax in relapsed chronic lymphocytic leukemia, N Eng/ J Med, 2016;374:311-22.

41. Stilgenbauer S, Eichhorst B, Schetelig J, et al., Venetoclax in relapsed or refractory chronic lymphocytic leukaemia with 17p deletion: a multicentre, open-label, phase 2 study, Lancet Oncol, 2016:17:768-78

42. Cervantes-Gomez F, Lamothe B, Woyach JA, et al., Pharmacological and protein profiling suggests venetoclax (ABT-199) as optimal partner with ibrutinib in chronic lymphocytic leukemia, Clin Cancer Res, 2015;21:3705-15

43. Herman SEM, Gordon AL, Wagner AJ, et al., Phosphatidylinositol 3-kinase- $\delta$ inhibitor CAL-101 shows promising preclinical activity in chronic lymphocytic leukemia by antagonizing intrinsic and extrinsic cellular survival signals, Blood, 2010;116:2078-88.

44. Furman RR, Sharman JP, Coutre SE, et al., Idelalisib and rituximab in relapsed chronic lymphocytic leukemia, N Engl J Med, 2014:370:997-1007.

45. O'Brien S, Patel M, Kahl BS, et al., Duvelisib (IPI-145), a PI3K- $\delta$, $\gamma$ inhibitor, is clinically active in patients with relapsed/refractory chronic lymphocytic leukemia, Blood, 2014;124:3334.

46. Balakrishnan K, Peluso M, Fu M, et al., The phosphoinositide3-kinase (PI3K)-delta and gamma inhibitor, IPI-145 (Duvelisib), overcomes signals from the PI3K/AKT/S6 pathway and promotes apoptosis in CLL, Leukemia, 2015:29:1811-22.

47. Dong S, Guinn D, Dubovsky JA, et al., IPI-145 antagonizes intrinsic and extrinsic survival signals in chronic lymphocytic leukemia cells, Blood, 2014;124:3583-6.

48. Porcu P, Flinn I, Kahl BS, et al., Clinical activity of duvelisib (IPI-145), a phosphoinositide-3-kinase- $\delta$, $\gamma$ inhibitor, in patients previously treated with ibrutinib, Blood, 2014;124:3335.

49. Burris HA, Patel MR, Brander DM, et al., TGR-1202, a novel once daily PI3KS inhibitor, demonstrates clinical activity with a favorable safety profile, lacking hepatotoxicity, in patients with chronic lymphocytic leukemia and B-cell lymphoma, Blood, 2014;124:1984.

50. Lunning MA, Vose J, Fowler N, et al., Ublituximab+ TGR-1202 Demonstrates Activity and a Favorable Safety Profile in Relapsed/ Refractory B-Cell NHL and High-Risk CLL: Phase I Results, Blood, 2015;126:1538

51. Chanan-Khan A, Miller KC, Musial L, et al., Clinical efficacy of lenalidomide in patients with relapsed or refractory chronic Iymphocytic leukemia: results of a phase II study, I Clin Oncol, 2006;24:5343-9.

52. Ferrajoli A, Lee B-N, Schlette EJ, et al., Lenalidomide induces complete and partial remissions in patients with relapsed and refractory chronic lymphocytic leukemia, Blood, 2008;111:5291-7

53. Salihoglu A, Ar MC, Soysal T, Novelties in the management of B-cell malignancies: B-cell receptor signaling inhibitors and Ienalidomide, Expert Rev Hematol, 2015;8:765-83.

54. Badoux XC, Keating MJ, Wen S, et al., Lenalidomide as initial therapy of elderly patients with chronic lymphocytic leukemia, Blood, 2011;118:3489-98.

55. Chen $\mathrm{Cl}$, Paul $\mathrm{H}$, Wang T, et al., Long-term follow-up of a phase 2 trial of single agent lenalidomide in previously untreated patients with chronic lymphocytic leukaemia, Br J Haematol, 2014; 165:731-3.

56. Chen $\mathrm{Cl}$, Bergsagel $\mathrm{PL}$, Paul $\mathrm{H}$, et al., Single-agent lenalidomide in the treatment of previously untreated chronic lymphocytic leukemia, J Clin Oncol, 2010;29:1175-81.

57. Chanan-Khan A, Miller KC, Lawrence D, Padmanabhan S, Miller A, Hernandez-lllatazurri F, et al. Tumor flare reaction associated with lenalidomide treatment in patients with chronic lymphocytic leukemia predicts clinical response, Cancer, 2011:117:2127-35.

58. Fink AM, Bahlo J, Sandra $R$, et al., Lenalidomide Maintenance after Front Line Therapy Substantially Prolongs Progression Free Survival in High Risk CLL: Interim Results of a Phase 3 Study (CLL M1 study of the German CLL Study Group), Presented at: 58th Annual Meeting of the American Society of Hematology, San Diego US, December 3, 2016.

59. Ramsay AG, Clear AJ, Fatah R, Gribben JG, Multiple inhibitory ligands induce impaired T-cell immunologic synapse function in chronic lymphocytic leukemia that can be blocked with Ienalidomide: establishing a reversible immune evasion 
mechanism in human cancer, Blood, 2012;120:1412-21.

60. Kater AP, Tonino SH, Egle A, Ramsay AG, How does lenalidomide target the chronic lymphocytic leukemia microenvironment? Blood, 2014:124:2184-9.

61. Brusa D, Serra S, Coscia M, et al., The PD-1/PD-L1 axis contributes to T-cell dysfunction in chronic lymphocytic leukemia Haematologica, 2013:98:953-63.

62. Riches JC, Davies JK, MCClanahan F, et al., T cells from CLL patients exhibit features of T-cell exhaustion but retain capacity for cytokine production, Blood, 2013;121:1612-21.

63. Jain N, Basu S, Thompson PA, et al., Nivolumab Combined with Ibrutinib for CLL and Richter Transformation: A Phase || Trial. Presented at: 58th Annual Meeting of the American Society of Hematology, San Diego US, December 3,2016.

64. Porter DL, Hwang W-T, Frey NV, et al., Chimeric antigen receptor $\mathrm{T}$ cells persist and induce sustained remissions in relapsed refractory chronic lymphocytic leukemia, Sci Trans/ Med 2015; 7:303ra139.

65. Mato $\mathrm{A}$, Porter $\mathrm{DL}, \mathrm{A}$ drive through cellular therapy for $\mathrm{CLL}$ in 2015: allogeneic cell transplantation and CARs, Blood, 2015; 126:478-85.

66. Buchner M, Baer C, Prinz G, et al., Spleen tyrosine kinase inhibition prevents chemokine- and integrin-mediated stroma protective effects in chronic lymphocytic leukemia, Blood 2010;115:4497-506.

67. Lapalombella R, Sun Q, Williams K, et al., Selective inhibitors of nuclear export show that CRM1/XPO1 is a target in chronic lymphocytic leukemia, Blood, 2012;120:4621-34.

68. Quiroga MP, Balakrishnan K, Kurtova AV, et al., B-cell antigen receptor signaling enhances chronic lymphocytic leukemia cell migration and survival: specific targeting with a novel spleen tyrosine kinase inhibitor, R406, Blood, 2009:114:1029-37.

69. Suljagic M, Longo PG, Bennardo $S$, et al., The Syk inhibito fostamatinib disodium (R788) inhibits tumor growth in the Emu- TCL1 transgenic mouse model of CLL by blocking antigen- dependent B-cell receptor signaling, Blood, 2010;116:4894-905

70. Ward E, Mittereder N, Kuta E, et al., A glycoengineered anti-CD19 antibody with potent antibody-dependent cellular cytotoxicity activity in vitro and lymphoma growth inhibition in vivo Br J Haematol, 2011:155:426-37.

71. Herbst $R$, Wang $Y$, Gallagher $S$, et al., B-cell depletion in vitro and in vivo with an afucosylated anti-CD19 antibody, J Pharmacol Exp Ther, 2010:335:213-22.

72. Herman SE, Barr PM, MCAuley EM, et al., Fostamatinit inhibits B-cell receptor signaling, cellular activation and tumor proliferation in patients with relapsed and refractory chronic lymphocytic leukemia, Leukemia, 2013;27:1769-73.

73. Zhong Y, El-Gamal D, Dubovsky JA, et al., Selinexor suppresses downstream effectors of B-cell activation, proliferation and migration in chronic lymphocytic leukemia cells, Leukemia, 2014;28:1158-63.

74. Robak T, Robak P, Anti-CD37 antibodies for chronic lymphocytic leukemia, Expert Opin Biol Ther, 2014;14:651-61.

75. Currie KS, Kropf JE, Lee T, et al., Discovery of GS-9973, a selective and orally efficacious inhibitor of spleen tyrosine kinase, J Med Chem, 2014; 5:3856-73.

76. Sharman J, Hawkins M, Kolibaba K, et al., An open-label phase 2 trial of entospletinib (GS-9973), a selective spleen tyrosine kinase inhibitor, in chronic lymphocytic leukemia, Blood, 2015; 125:2336-43.

77. Maddocks KJ, Pagel J, Byrd JC, et al., Phase 1b study of otlertuzumab (TRU-016), an Anti-CD37 ADAPTIRTM protein, in combination with rituximab in patients with chronic lymphocytic leukemia (CLL), Blood, 2014;124:4671.

78. Byrd JC, Pagel JM, Awan FT, et al., A phase 1 study evaluating the safety and tolerability of otlertuzumab, an anti-CD37 monospecific ADAPTIR therapeutic protein in chronic lymphocytic leukemia, Blood, 2014;123:1302-8.

79. Beckwith KA, Frissora FW, Stefanovski MR, et al,. The CD37targeted antibody-drug conjugate IMGN529 is highly active against human CLL and in a novel CD37 transgenic murine leukemia model, Leukemia, 2014;28:1501-10.

80. Heider KH, Kiefer $\mathrm{K}$, Zenz $\mathrm{T}$, et al., A novel Fc-engineered monoclonal antibody to CD37 with enhanced ADCC and high proapoptotic activity for treatment of B-cell malignancies, Blood, 2011:118:4159-68.

81. Awan $F T$, Hillmen $P$, Hellmann $A$, et al., A randomized, open-label, multicentre, phase $2 / 3$ study to evaluate the safety and efficacy of lumiliximab in combination with fludarabine, cyclophosphamide and rituximab versus fludarabine, cyclophosphamide and rituximab alone in subjects with relapsed chronic lymphocytic leukaemia, Br J Haemato , 2014;167:466-77.

82. Woyach JA, Awan F, Flinn IW, et al., A phase 1 trial of the FCengineered CD19 antibody XmAb5574 (MOR00208) demonstrates safety and preliminary efficacy in relapsed CLL, Blood, 2014; 124:3553-60

83. Hing ZA, Mantel R, Beckwith KA, et al., Selinexor is effective in acquired resistance to ibrutinib and synergizes with ibrutinib in chronic lymphocytic leukemia, Blood, 2015;125:3128-32.

84. Andritsos L, Byrd JC, Jones JA, et al., Preliminary results from a phase I dose escalation study to determine the maximum tolerated dose of plerixafor in combination with rituximab in patients with relapsed chronic lymphocytic leukemia, Blood 2010;116:2450

85. Alsagaby SA, Brennan P, Pepper C, Key Molecular Drivers of Chronic Lymphocytic Leukemia, Clin Lymphoma Myeloma Leuk 2016;16:593-606.

86. Jain $\mathrm{N}$, Chen $\mathrm{Q}$, Ayer $\mathrm{T}$, et al., Prevalence and Economic Burden of Chronic Lymphocytic Leukemia (CLL) in the Era of Oral Targeted Therapies, Blood, 2015:126:871.

87. Shanafelt TD, Borah BJ, Finnes HD, et al., Impact of ibrutinib and idelalisib on the pharmaceutical cost of treating chronic lymphocytic leukemia at the individual and societal levels, J Oncol Pract, 2015;11:252-8. 